\title{
EVALUACIÓN DEL ALMIDÓN DE PAPA COMO FLOCULANTE PARA EL TRATAMIENTO DE AGUAS RESIDUALES DOMESTICAS
}

\section{EVALUATION OF POTATO STARCH AS FLOCCULANT FOR THE TREATMENT OF DOMESTIC SEWAGE}

\author{
Herrera $\mathbf{M}^{*}$. \\ Universidad Pedagógica y Tecnológica de Colombia, Facultad de Ingeniería, \\ Programa de Maestría en Ingeniería Ambiental \\ Avenida Central del Norte 39-115 Tunia-Colombia. Correo electrónico: mahe1008@yahoo.es
}

Recibido 23 de Julio 2015; aceptado 25 de octubre de 2015

\section{RESUMEN}

Siendo el agua uno de los recursos primordiales para la existencia de los seres vivos, motivo por el cual el hombre se preocupa por tratar de mantener esté lo menos contaminado posible, se genera la necesidad de realizar tratamientos para su clarificación, mediante la investigación de alternativas para los insumos químicos, los cuales permitan mejorar la calidad del agua, reducir los impactos ambientales $y$ posteriormente realizar un reusó. Para lo cual se evaluó el proceso de coagulación del agua residual, mediante la adición de un coagulante-floculante natural obtenido del almidón de papa para el tratamiento de aguas residuales, debido a que los polímeros orgánicos a diferencia de los sintéticos usados generalmente en las plantas de tratamiento convencionales presentan la mínima o nula toxicidad, son menos perjudiciales, ambientalmente inocuos para remover la turbidez de las aguas, sumado a la capacidad que tiene el departamento Boyacense de ser uno de los mayores productores de tubérculos de la mejor calidad, para lo cual 
se obtuvo Almidón Nativo y se modificó químicamente mediante oxidación, se caracterizó por FTIR y MEB, se realizaron ensayos de simulación para determinar la concentración y dosis óptima y se evaluó su turbiedad y color. Los resultados señalan que el almidón de papa, como alternativa técnica de coagulación - floculación es buena, debido a que es una opción de tecnología adaptable, viable y flexible a las condiciones de operación de las PTAR convencionales para posteriormente realizar un adecuado $y$ seguro reusó destinado a otros fines.

Autor a quien dirigirse la correspondencia. Correo electrónico: mahe1008@yahoo.es
Palabras clave: Almidón, floculación, solanum tuberosum, tratamiento de aguas.

\section{ABSTRACT}

Since water is a primary resource for the existence of living beings, why the man cares about trying to maintain is least polluted possible, the need for treatments for clarification is generated by researching alternatives for chemical inputs, which will improve water quality, reduce environmental impacts and then perform reuse. For which the coagulation process wastewater was evaluated by adding a coagulantflocculant Natural potato starch obtained for wastewater treatment, because unlike organic polymers generally used in synthetic plants conventional treatment have minimal or no toxicity, are less harmful, environmentally safe to remove the turbidity of the water, combined with the ability of the Boyacense department being one of the largest producers of tubers of better quality, for which Native starch was obtained and was chemically modified by oxidation, was characterized by FTIR and MEB, simulation tests were conducted to 
determine the optimal dose and concentration, and turbidity and color was evaluated. The results indicate that potato starch as alternative technique of coagulation - flocculation is good, because it's a convenient and customizable, viable and flexible operating conditions of conventional PTAR later to make appropriate technology and safe reuse intended other purposes.

Keywords: starch, flocculation, solanum tuberosum, water treatment.

\section{INTRODUCCIÓN}

La contaminación de las aguas es una de las causas de millones de muertes cada año en el mundo, por lo cual se hace necesario someterla a una serie de tratamientos previos antes de ser utilizada o reutilizada, para así garantizar la remoción total 0 parcial de dichas impurezas, esto mediante métodos elementales los cuales incluyen la clarificación, floculación y sedimentación. La clarificación es una etapa importante dentro del proceso de tratamiento de aguas, que incluye proceso de coagulación-floculación en el cual las partículas presentes en el agua se aglomeran formando pequeños gránulos con un peso específico mayor; de esta forma las partículas sedimentan y ocurre la remoción de los materiales en suspensión, lo que permite que el agua alcance las características físicas y organolépticas idóneas, las cuales garantiza la eliminación de contaminantes según las normas y estándares nacionales e internacionales de salud pública (Gonzales et al ., 2006).

Generalmente las plantas de tratamiento de aguas convencionales utilizan como coagulantes las sales de aluminio o de hierro para desestabilizar partículas coloidales, pero desde los años 70 en adelante se propuso la utilización de coagulantes naturales de cada región, con el propósito de reducir el consumo de reactivos químicos; sin embargo, el 
desarrollo de estos coagulantes no tuvo un auge significativo debido a la comercialización y utilización de polímeros sintéticos en procesos convencionales de tratamiento como coagulantes primarios 0 ayudantes de floculación, pero los cuales pueden presentar a largo plazo una serie de problemas, entre los cuales se encuentra; niveles demasiado altos de monómeros residuales en los productos finales, elevada densidad de carga iónica (Ochoa, 2003), persistencia en el medio durante muchos años, ocupando espacio en los rellenos sanitarios y causando daño al ambiente por la emisión de gases si se les incinera sin los cuidados necesarios; generando de esta manera sustancias peligrosas y efectos secundarios en la salud humana principalmente enfermedades neurológicas como Alzheimer (Molano, 2011), o pudiendo no ser removidas durante el tratamiento convencional antes de que estas sean reusadas por parte del usuario (Rodríguez, 2007).

Teniendo en cuenta esta situación, día tras día se busca desarrollar e implementar nuevas tecnologías con las cuales se puedan llevar a cabo estos procesos de forma más económica, eficiente y amigable con el medio ambiente. La desestabilización o coagulación del agua, ha sido siempre considerada una de las etapas más relevantes del tratamiento, por ello se le ha dado mucha importancia a los agentes coagulantes utilizados, ya que sin ellos, esta fase fundamental no sería posible. En la actualidad los coagulantes preferidos siguen siendo las sales minerales de hierro y aluminio, sin embargo, los polielectrólitos orgánicos cada día adquieren mayor importancia (Singley, 1986).

Los polímeros orgánicos o de origen natural presentan diferentes propiedades entre las cuales se encuentra la alta viabilidad para formar geles, debido a su compleja estructura molecular y a su gran capacidad de hidratación (hasta un $80 \%$ de incremento en peso); alta idoneidad complejante que es la responsable de la retención de iones metálicos; gran potencial biodegradante, a diferencia de muchos compuestos coagulantes sintéticos; y mínima toxicidad en polímeros naturales por lo que se les usa en muchos casos como alimentos o aditivos en diversos productos. De acuerdo con lo anterior, las alternativas de tratamiento de aguas basadas en la utilización de coagulantes naturales en los procesos de clarificación, se hace más que necesaria, imprescindible teniendo en cuenta las condiciones económicas actuales de muchos países (Vázquez, 1994). 
La presente investigación persigue demostrar que con el uso y modificación del almidón nativo de la especie de papa (Solanum tuberosum) proveniente del Departamento de Boyacá, se cuenta con la capacidad para la remoción de turbidez de aguas contaminadas y una posible alternativa y sustituto de los polímeros sintéticos debido a su alto costo.

\section{MATERIALES Y MÉTODOS}

La presente investigación se llevó a cabo en las instalaciones de la Universidad Pedagógica y Tecnológica de Colombia, en el Laboratorio de Investigación en Química y Tecnología de Alimentos. Se divide en cuatro fases:

\section{Fase I: Extracción y caracterización del} almidón.

Para evitar que la materia prima a utilizar sufra algún tipo de deterioro fisiológico a causa de la acumulación de compuestos fenólicos, microbiológicos y/o mecánicos, se da el uso adecuado en un tiempo inferior a 24 horas, luego de su cosecha. La fase de extracción se desarrolló mediante el siguiente procedimiento (Ver figura 1).

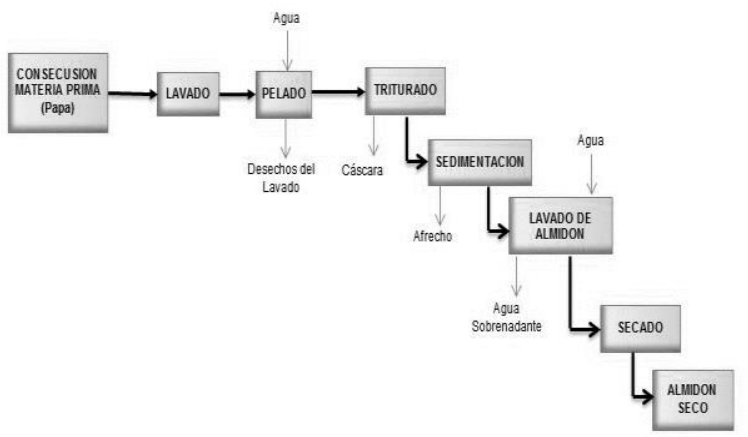

Figura 1. Etapas de la extracción del almidón de papa.

Se desarrolló un control de calidad de la materia prima mediante un análisis básico de humedad, fibra y cenizas por métodos gravimétricos y colorimétricos de acuerdo a las normas del AOAC (Association of Analytical Communities).

Obtenido el almidón se realizaron los cálculos necesarios para determinar el porcentaje de rendimiento.

\section{Fase II: Modificación química del almidón por oxidación.}

El almidón oxidado $(A O)$ se obtuvo en el laboratorio siguiendo la metodología propuesta por Wang y Wang, con algunas modificaciones. Se preparó una suspensión de almidón al $20 \%$ y se agito constantemente a $35^{\circ} \mathrm{C}$ por 15 minutos. Se ajustó el pH con $\mathrm{NaOH} 0.1 \mathrm{~N}$ y se adiciono $\mathrm{NaOCl}$ al $2.0 \%$ de cloro activo $(\mathrm{p} / \mathrm{v})$, manteniendo el $\mathrm{pH}$ a 9,5. La reacción se 
dejó por una hora adicional. Se neutralizó a $\mathrm{pH} 7$ con $\mathrm{HCl} 0.1 \mathrm{~N}$. Se lavó con agua destilada dos veces y se secó en estufa a $35^{\circ} \mathrm{C}$ por 48 horas y el almidón obtenido se molió y tamizó.

Fase III: Identificación estructural mediante análisis instrumental.

Análisis de Espectroscopia Infrarroja FTIR: Los espectros IR del AN y AO se obtuvieron con un equipo IRPrestige-21 marca SHIMADZU.

Análisis de microscopia electrónica de barrido (MEB): La morfología de los AN y $A O$ se observaron en un equipo LEO-430. Las muestras se recubrieron con una película delgada de carbono en vacío y se tomaron micrografías a 2000x.

\section{Fase IV: Prueba de jarras}

La Prueba de Jarras es la técnica más ampliamente usada para determinar la dosis óptima o mejor dosis de químicos para procesos de coagulación-floculación a nivel de laboratorio. (Ver figura 2).

Se tomó como fuente de abastecimiento, el agua residual procedente del Municipio de Paipa a la altura de la Central Termoeléctrica, la cual mantuvo una turbiedad en promedio de 355 UNT, Color 1660 UPC y pH de 7.34. La determinación de turbiedad, color y pH se realizó según los métodos normalizados del APHA/AWWA/ número 2130 y 2120 respectivamente. El ensayo de jarras tuvo las siguientes consideraciones técnicas:

1) La solución patrón para el sulfato de aluminio tipo $\mathrm{B}$, fue del $1 \%$ y la de los almidones de papa del 0,5\%.

2) El mezclado rápido se realizó a 120 RPM por un minuto.

3) Para la operación de floculación se utilizó un tiempo de 20 min a velocidad de 30 RPM.

4) En la sedimentación, el tiempo de decantación fue de 20 minutos, sin gradiente de velocidad.

5) Para la determinación de la dosis del Sulfato de Aluminio, se tomó en referencia teniendo en cuenta las dosis reportadas en la mayoría de los estudios consultados, las cuales por lo general se encontraban en el intervalo de 30 a 60 mg/L.

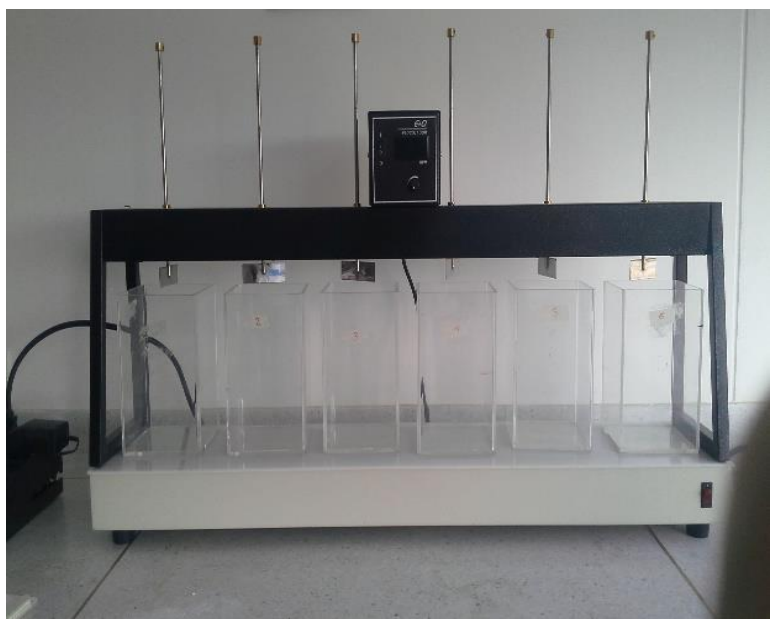

Figura 2. Ensayo prueba de Jarras. 


\section{RESULTADOS Y DISCUSIÓN}

El almidón obtenido es un polvo fino, blanco, inodoro (ver Figura 3), insípido produce ligera crepitación cuando es comprimido entre los dedos.

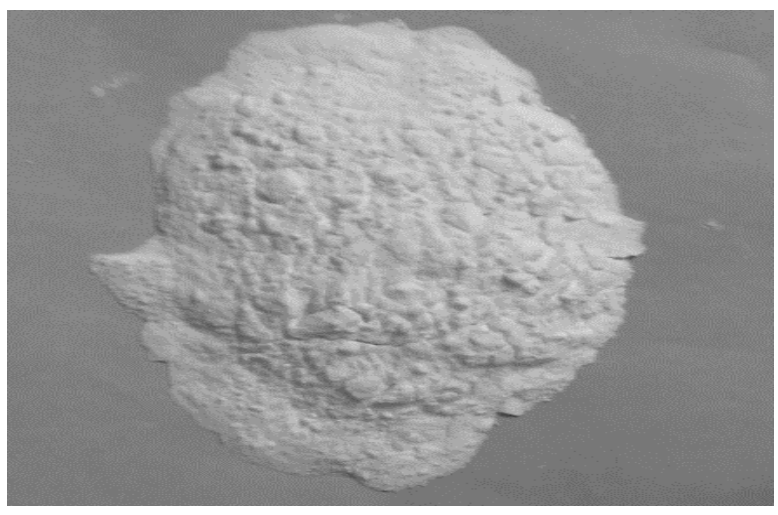

Figura 3. Almidón de papa seco

\section{Caracterización del almidón:}

La Tabla 1, presenta las características químicas del almidón de papa, de acuerdo a los datos obtenidos en la caracterización del almidón nativo se encontró un valor de humedad del $18.10 \%$ siendo un resultado mayor con respecto a la teoría (Arguello et al., 2004), por lo cual se aumenta el riesgo a generar actividad de agua en el almacenamiento del almidón y por ende a un posible ataque de microorganismos. Mientras que la ceniza y la fibra disminuyen notoriamente, debido a que en el proceso de extracción y purificación del almidón dichos elementos fueron retirados en su mayoría. Básicamente estos porcentajes bajos determinan la eficiencia en el proceso de extracción.

Tabla 1. Caracterización del almidón

\begin{tabular}{ccc}
\hline Variable & $\begin{array}{c}\text { Almidón de } \\
\text { Papa }\end{array}$ & Teórico \\
\hline Humedad & $18,10 \%$ & $13,10 \%$ \\
Cenizas & $0,23 \%$ & $0,2863 \%$ \\
Fibra & $0,0093 \%$ & $0,2 \%$ \\
\hline
\end{tabular}

\section{Porcentaje rendimiento de almidón:}

La siguiente Tabla, muestra el porcentaje de rendimiento del almidón de papa (Ver Tabla 2).

Tabla 2. Porcentaje de rendimiento

\begin{tabular}{lcc}
\hline \multicolumn{1}{c}{ Material } & Cantidad & $\begin{array}{c}\text { \% Rendimiento } \\
\text { Teórico }\end{array}$ \\
\hline Peso inicial $(\mathrm{g})$ & 1000 & \\
Pérdida o & 81,21 & $12-19$ \\
Regazo (\%) & 18,79 & \\
\hline Almidón (\%) & & \\
\hline
\end{tabular}

El porcentaje de rendimiento obtenido de la especie de papa, se encuentra dentro del rango promedio de los parámetros teóricos (Mora y Pita, 2006). Sin embargo, cabe resaltar que en el proceso de pelado y lavado hay perdida de almidón. Este porcentaje de rendimiento fue favorable, debido al proceso de extracción satisfactorio. 


\section{Análisis de Espectroscopia Infrarroja (FTIR):}

En la Figura 4, se presenta el espectro FTIR del AN el cual presenta las bandas características para este tipo de compuesto. La vibración del grupo hidroxilo aparece como una banda ancha en $3273 \mathrm{~cm}-1$, la vibración del enlace C-O de hidroxilos secundarios aparece en $995 \mathrm{~cm}-1$ y la vibración C-O-C en $1149 \mathrm{~cm}-1$, las bandas de vibraciones de tensión en el plano correspondientes a $\mathrm{OH}$, se localizan en $1336 \mathrm{~cm}-1$ y la banda de tensión fuera del plano correspondiente a $\mathrm{CH}$ se encuentra en 1076 cm-1. Confirmándose de esta manera la molécula de glucosa presente en el almidón nativo extraído de la papa.

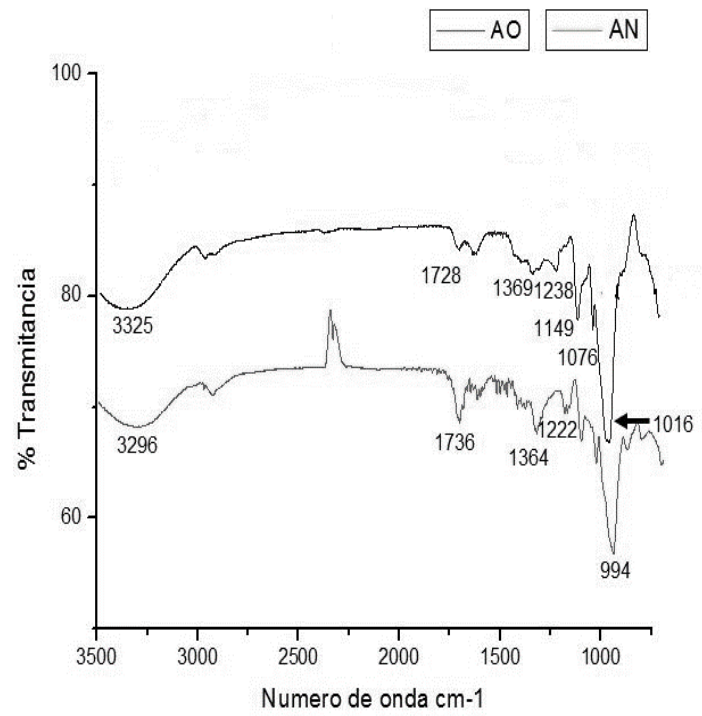

Figura 4. Espectros FTIR, Almidón Nativo y Oxidado.

Se presenta el espectro FTIR del AO en el cual aparece una nueva banda a $1736 \mathrm{~cm}-1$ que se atribuye a la vibración del grupo carbonilo formado por la reacción de oxidación. Se observan dos nuevas bandas en $1364 \quad \mathrm{~cm}-1 \quad$ y $1222 \quad \mathrm{~cm}-1$ correspondientes a las tensiones de los enlaces $\mathrm{C}-\mathrm{O}-\mathrm{H}$ y C-O respectivamente, de los grupos carboxilos que también se forman durante la oxidación del almidón. Lo anterior denota claramente una modificación química del almidón (Ver figura 4).

\section{Análisis de microscopia electrónica de barrido (MEB) de los almidones:}

Al ser observadas las micrografías, (Ver figura 5), se tiene que los gránulos del Almidón Nativo presentan formas circulares para los tamaños pequeños y elípticos para los tamaños grandes.

Se observó que el almidón de papa muestra una distribución normal de tamaño, este rango de distribución va de 5 a $40 \mu \mathrm{m}$, lo que significa que dichos almidones poseen gránulos pequeños, medianos y grandes (Robles, 2012). En el AN Se logra evidenciar claramente la forma de los granos y una disposición uniforme. Mientras que en el $\mathrm{AO}$, (Ver figura 6), muchos de los gránulos han perdido su forma, además que se inicia la formación de aglomerados indicando de esta manera una fuerte unión entre los puentes de hidrógenos de cada una de las cadenas del polímero. 


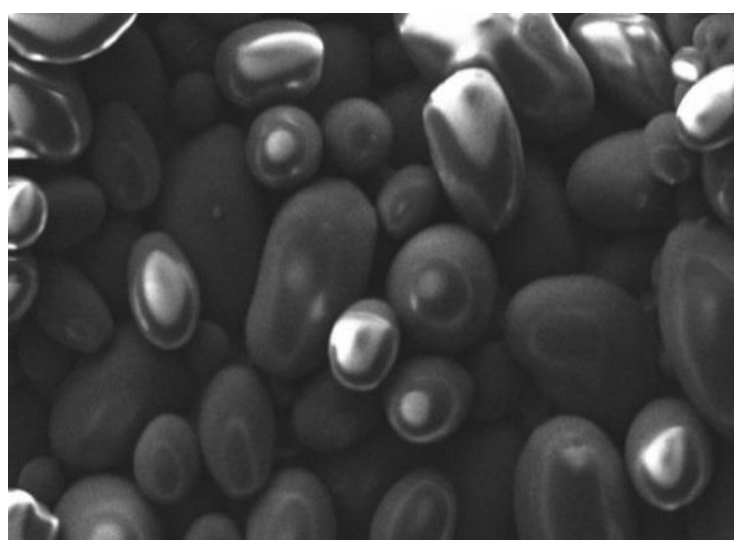

Figura 5. Fotografía MEB Almidón Nativo

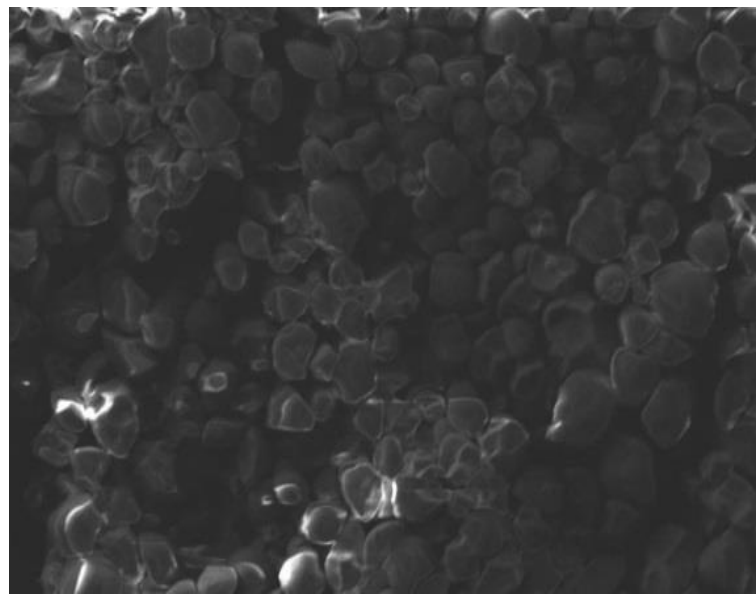

Figura 6. Fotografía MEB Almidón Oxidado

\section{Prueba de Jarras}

Las características fisicoquímicas iniciales de la muestra de agua objeto del estudio se presentan en la Tabla 3.

Tabla 3. Características Iniciales del Agua residual

\begin{tabular}{ll}
\hline Parámetro & Valor \\
\hline Turbiedad (NTU) & 355 \\
Color (Pt-Co) & 1660 \\
$\mathrm{pH}$ & 7,34 \\
\hline
\end{tabular}

El coagulante en polvo (Almidón) antes de ser adicionado a las muestras de agua cruda, se solubilizó en agua destilada, para lograrlo se agito durante 15 minutos a 250 rpm, manteniendo una temperatura de 35 ${ }^{\circ} \mathrm{C}$.

En las figuras 7 a 9 se presentan los valores de la turbiedad y el color del agua tratada con los coagulantes estudiados; sulfato de aluminio, almidón nativo de papa (AN) y almidón oxidado de papa (AO).

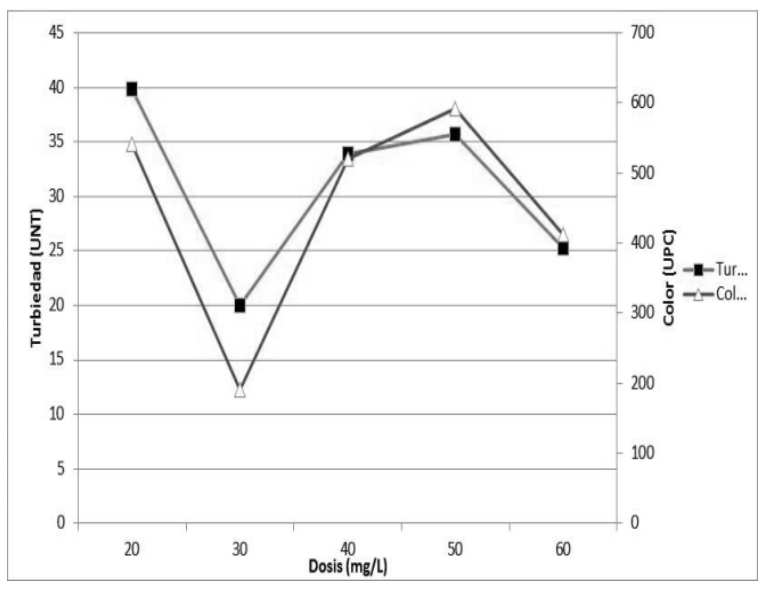

Figura 7. Comportamiento del Sulfato de Aluminio.

Se observa que el coagulante utilizado tradicionalmente (Sulfato de Aluminio) para una dosis optima $30 \mathrm{mg} / \mathrm{L}$, genera rendimientos de turbiedad próximos a los 20 UNT, color sobre los 190.5 UPC y un pH de 7,43 (Ver figura 7). Lo cual indica que continúa siendo un agua con un color residual alto y que no se lograr alcanzar 
valores cercanos a los expuestos por el decreto 1594 de 1984.

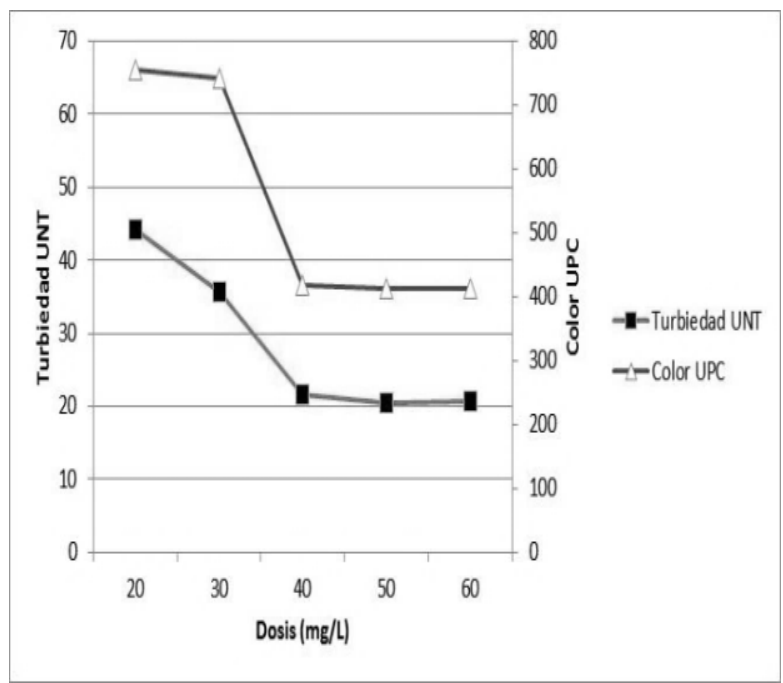

Figura 8. Comportamiento del AN

En términos de la utilización del almidón nativo de papa para la remoción de color y turbidez, se observa (Ver figura 8) que al aplicar este coagulante en una dosis optima de $50 \mathrm{mg} / \mathrm{L}$, conduce a aportar beneficios apreciables en la calidad del agua tratada, arrojando valores de turbiedad de 20.4 UNT, color de 412 UPC y pH 7,56, valores próximos a los encontrados con el uso del sulfato de aluminio, exceptuando el valor de color que fue aproximadamente 2 veces más alto que el anterior.

Lo cual representa ser muy favorable en la operación del tratamiento de aguas residuales.

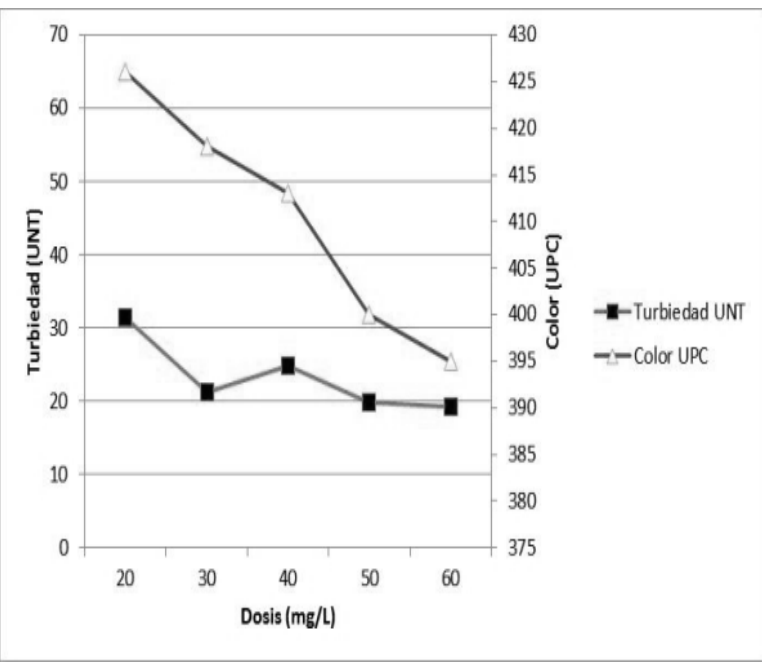

Figura 9. Comportamiento del AO.

En la Figura anterior se observa que el color tiende a reducir a medida que disminuye de la misma manera la turbidez, la dosis optima se encontró al aplicar $60 \mathrm{mg} / \mathrm{L}$, los resultados de turbiedad son de 19,2 UNT, color de 395 UPC y pH 7,05, lo cual indica que los valores se encuentran similares y próximos a los hallados con la utilización de alumbre y del AN.

De acuerdo a lo anterior, teniendo en cuenta los valores reportados de turbiedad, se observó que el almidón de papa nativo presenta los mejores resultados al ser usado como coagulante-floculante, generando una aglutinación mejor de las partículas suspendidas.

En cuanto a la variable del color, se observa que los valores disminuyen gradualmente, sin embargo, continúan siendo altos, por lo 
cual no permiten el cumplimiento del Decreto Colombiano 1594 de 1984, por parte de ninguno de los floculantes usados anteriormente.

El uso de polímeros orgánicos provenientes del almidón de papa, ya sea nativo o modificado químicamente por oxidación, han demostrado mejorar la calidad del efluente e incrementa la velocidad de sedimentación, por lo cual al utilizar como coagulante natural al almidón de papa se reduciría el volumen de lodos depositados, de esta manera se facilita la disposición final y la reducción de costos de tratamiento.

El almidón de papa, puede ser utilizado como una alternativa para la remoción de carga contaminante en los cuerpos de agua, tanto en centros urbanos como rurales, garantizando su reusó y reduciendo el riesgo potencial a la salud y la eutrofización de las aguas.

\section{CONCLUSIONES}

La mayoría de plantas de tratamiento de aguas residuales municipales en Colombia usan generalmente sales de aluminio o de hierro, en muy pocos casos optan por utilizar polímeros naturales para la desestabilización de partículas coloidales presentes en los efluentes, debido principalmente al desconocimiento que existe por las ventajas y cualidades que cuentan estos productos naturales en el tratamiento de las aguas contaminadas.

Los resultados experimentales establecidos en condiciones de ensayo, reportó que el mejor coagulante-floculante fue el almidón de papa nativo ya que arroja datos muy próximos a los hallados con el sulfato de aluminio, sin embargo, la oxidación del almidón indica que no es la modificación adecuada ya que con esta se consigue una aglomeración de los gránulos y por ende se dificulta una buena realización del proceso de coagulación-floculación.

Este tipo de coagulante-floculante natural surte efectos positivos en la remoción de partículas coloidales sin alterar el $\mathrm{pH}$ del agua después del tratamiento, sus lodos son inocuos y de menor cantidad que la coagulación con sulfato de aluminio.

Se recomienda que estos ensayos con polímeros naturales sean implementados bajo diferentes condiciones como el tipo de agua, ya sea la superficial, subterránea o la destinada a la potabilización, con el 
propósito de abarcar mayores usos de estos coagulantes, mejorar las condiciones de salubridad, los costos, minimizar el impacto ambiental de los cuerpos de agua y verificar la eficiencia y eficacia de estos en diferentes condiciones de operación.

Lo anterior muestra una alternativa viable para reemplazar el uso del sulfato de aluminio inicialmente en zonas urbanas y periurbanas donde no es posible hacer llegar el producto coagulante. Esta acción disminuirá las enfermedades de origen hídrico como las enfermedades diarreicas agudas (EDA), y consecuentemente se traduce en ahorro en medicamentos y costos hospitalarios, así como el mejoramiento de la calidad del agua, en este caso para fines de reusó.

\section{REFERENCIAS BIBLIOGRÁFICAS}

APHA, AWWA, WPCP. Métodos normalizados para el análisis de aguas potables y residuales. 2000.

Arguello, M. Dueñas Y. \& Rojas D. (2004) Extracción y tratamiento enzimático del almidón para la obtención de dextrinas y jarabe de glucosa. Trabajo de grado para optar al título de Químico en Alimentos. Tunja. 2004.

Gonzales, Griselda, et al. (2006). Uso del exudado gomoso por Samanea saman en la potabilización de las aguas. Revista Técnica de la Facultad de Ingeniería Universidad del Zulia. Vol 29, 226 - 231. 2006.

Molano, L. (2011). Las semillas de Moringa Oleífera Lam. Como alternativa coagulante natural para la purificación de agua. Tesis de especialista, Universidad Industrial de Santander, Bucaramanga, Colombia. 2011.

Mora M. Y Pita Y. (2006). Extracción y caracterización fisicoquímica y funcional del almidón de malanga, cultivada en el Municipio de Miraflores. Trabajo de grado para optar al título de Químico de Alimentos. Tunja. 2006.

Ochoa, J. Obtención de floculantes no iónicos y aniónicos (densidad de carga 40\%) por polimerización en microemulsión inversa, influencia del sistema tensioactivo. Revista Iberoamericana de Polímeros. Vol 4, 1416. 2003. 
Robles M. (2012) Relación entre algunas propiedades fisicoquímicas y térmicas de gelatinización y retrogradación en almidón de papa nativa de Chiloé. Trabajo de grado para optar al título de Ingeniero en Alimentos. Valdivia. 2012.

Rodríguez, M. (2007). Evaluación del proceso de la coagulación para el diseño de una planta potabilizadora. Revista UMBRAL. Vol 20, 8-17. 2007.

Singley, J. (1986). Revisión de la teoría de coagulación del agua. Gainesville: Universidad de la Florida. 1986.

Vázquez, O. (1994). Extracción de coagulantes naturales del Nopal y aplicación en la clarificación de aguas superficiales. Tesis de especialista en Ingeniería Ambiental. Universidad Autónoma de Nuevo Colon, Monterrey, México. 1994.

Wang $Y$ and Wang L. (2003). "Physicochemical properties of common and waxy corn starches oxidized by different levels of sodium hypochlorite", Carbohydrate Polymers., vol. 52, pp. 207-217.2003. 